\title{
Peak-to-Average Power Reduction using Repeated Frequency Domain Filtering and Clipping in OFDM
}

\author{
Zainab Saad Hadi \\ University of Baghdad, College of Engineering \\ Electronic \& Communications Engineering \\ Department
}

\begin{abstract}
The Orthogonal Frequency Division Multiplexing is one of the widely used modulation techniques in the broadband wireless technology. One of the main problems of the OFDM is the high peak-to-average power ratio of transmitting signal due to the superposition of many subcarriers. This paper presents a new proposed peak-to-average power ratio reduction technique, which Repeated frequency domain filtering and clipping over LET channel and compare with Repeated clipping and frequency domain filtering (RCF) technique. The paper highlights the performance and advantages of the proposed technique. The simulations show that the proposed technique realizes an improved PAPR and BER.
\end{abstract}

\section{Keywords}

OFDM, PAPR, RFC

\section{INTRODUCTION}

During the last two decades, the demand for multimedia wireless communication services have grown tremendously and this trend are expected to continue in the near future. Orthogonal frequency division multiplexing (OFDM) is one of such multi-carrier techniques which have attracted vast research attention from academics, researchers and industries since last two decades. It has become part of new emerging standards for broadband wireless access [1]

Energy efficiency, particularly matters in future mobile communications networks. A key driving factor is the growing energy cost of network operation which can make up as much as $50 \%$ of the total operational cost nowadays [2]

The transmitted signal of OFDM exhibits a high Peak-ToAverage Power Ratio (PAPR). This high PAPR reduces the efficiency of high power amplifier and degrades the performance of the system.[3]

PAPR has a deleterious effect on battery lifetime in mobile applications. As handy devices have a finite battery life, it is significant to find ways of reducing the PAPR allowing for a smaller, more efficient HPA, which in turn will mean a longer lasting battery life.

In many low-cost applications, the problem of high PAPR may outweigh all the potential benefits of multicarrier transmission systems.[4]

\section{DEFINITIONS OF PAPR}

For a continuous time baseband OFDM signal, the PAPR of any signal is defined as the proportion of the maximum instantaneous power of the signal and its average power. If $x$ (t) is a transmitted baseband OFDM signal, then PAPR is defined as:

\author{
Buthaina Mosa Omran, Ph.D \\ University of Baghdad, College of Engineering \\ Electronic \& Communications Engineering \\ Department
}

$\operatorname{PAPR}[x(t)]=\frac{\left.\underset{0 \leq t \leq T_{s}}{\operatorname{MAX}}||_{x(t)} \mid{ }^{2}\right]}{p_{a v}}$

Where, $p_{a v}$ is the average power of $\mathrm{x}(\mathrm{t})$ and can be computed in frequency domain because IFFT is a unitary transformation $T_{S}$ is useful duration of an OFDM symbol [5].

\section{DISTRIBUTION OF PAPR}

To design and develop an effective PAPR reduction technique, it is very important to accurately identify the distribution of PAPR in OFDM systems. The distribution of PAPR plays an important role in the design of the whole OFDM system. The distribution of PAPR can be used in determining the proper output back-off of the HPA to minimize the total degradation. It can be used directly to calculate the BER and to estimate the achievable information rates [6].

The power of OFDM signal has chi-square distribution. The distribution of PAPR is often expressed on the one hand Complementary Cumulative Distribution Function (CCDF). In probability theory and statistics, the CCDF describes the probability that a real-valued random variable $\mathrm{X}$ with a given probability distribution will be found at a value greater than or equal to $\mathrm{x}[6]$.

The Cumulative Distribution Function (CDF) of the PAPR of the amplitude of a signal sample is given by

$F(z)=1-\exp (z)$

The CCDF of the PAPR of the data block is desired in our case is to compare outputs of different reduction techniques. This is given by:

$$
\begin{aligned}
& \operatorname{Pr}(P A P R>z)=1-\operatorname{Pr}(P A P R \leq z) \\
& =1-F(z)^{N} \\
& =1-\left(1-\exp (-z)^{N}\right.
\end{aligned}
$$

Where, $z$ is the given reference level.

\section{REPEATED CLIPPING AND FREQUENCY DOMAIN FILTERING (RCF)}

In the clipping technique hard limiting is applied to the amplitude of the complex values of the IFFT output. The filtering technique is designed to alleviate or cancel OOB distortion dependent on oversampling value but cannot correct in-band distortion. [7] .

The input vector $a_{0}, \ldots, a_{N-1}$ is first converted from the frequency to the time domain wing an oversize IFFT. $\mathrm{N}$ is the number of subcarriers in each OFDM symbol. For an oversampling factor of, the input vector is extended by adding 
$N(I-1)$ zeros; in the middle of the vector. This results in the trigonometric interpolation of the time domain signal [9].

Trigonometric interpolation gives perfect interpolation when the original signal consists of integral frequencies over the FFT window. This is the case for OFDM. The input of the Nyquist frequency, $N / 2$ has been omitted, as the interpolation technique does not work for this value [9]. This is not a practical limitation as all applications of OFDM null this input and most do not use a number of adjacent subcarriers. The interpolated signal is then clipped.

In this Technique hard-limiting is applied to the amplitude of the complex values of the IFFT output [10]

After an IFFT, the original signal is clipped in the time domain. The clipping can be described as shown below:

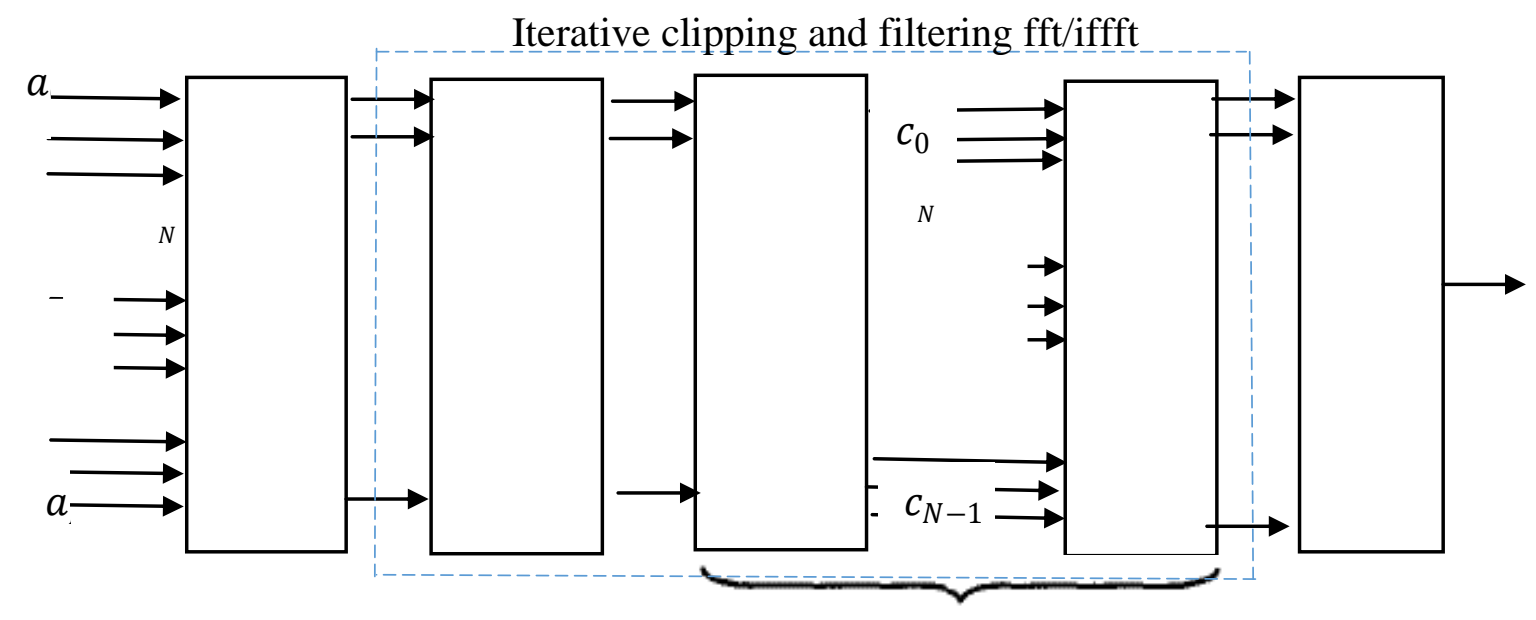

Figure 1 shows the block diagram of the new PAPR reduction scheme [8].

$$
C=\left\{\begin{array}{ll}
\sqrt{c_{R} * E\left[|x|^{2}\right]} * \frac{x}{|x|} & |x|^{2}>c_{m} \\
x & |x|^{2} \leq c_{m}
\end{array}\right\}
$$

Where $C$ represents the output of the time domain signal,

$c_{m}=c_{R} * E\left[|x|^{2}\right]$

, $c_{m}$ Is the threshold clipping level, signal power $=|x|^{2}$ $; E\left[|x|^{2}\right]$ Is the mean power. $c_{R}$ The clipping ratio is defined as the ratio of the clipping level to the mean power of the unclipped baseband signal.

As shown in the equation (6), the discrete time domain signal is clipped in the amplitude. At every point where the complex time domain signal exceeded the clipping level, the amplitude was reduced to the clipping level while the phase of the complex signal was unchanged [11].

The clipping is followed by frequency domain filtering to reduce OOB power caused by clipping. The filter consists of two FFT operations [10].

The clipped time domain signal $\mathrm{c}$ is then converted back into the discrete frequency domain using an FFT ,The inband discrete frequency components of the clipped signal $c_{0}, c_{\frac{N}{2}-1}, c_{N I_{1}-\frac{N}{2}+1}, \ldots, c_{N I_{1}-1}$ are passed unchanged to the inputs of the second IFFT while the OOB components, $c_{\frac{N}{2}+1}, \ldots, c_{N I_{1}-\frac{N}{2}}$ are nulled [12 and 13] this technique is repeated, depending on iteration number, usually choose between one and four. In this work has been selected four.

Although frequency domain filtering is a common signal processing technique the form shown in figure 1 is unusual. In most filtering applications the filter is designed to meet particular specifications in the continuous frequency domain. In this application, the wanted signal is an OFDM signal, which is the sum of discrete frequency components in each symbol period. The filter must therefore have as little effect as possible on the in-band discrete frequency domain while attenuating as much as possible any OOB components. This is precisely what is achieved by the simple filter structure in Fig 1 because the filter operates on a symbol by symbol basis; there is no filtering across symbol boundaries and so no resultant ISI. The filtering does cause some peak regrowth. However, this is much less than for clipping before interpolation [10, and 14]

The clipping noise is added at the transmitter rather than the receiver. In fading channels this means that in general the clipping noise will cause less degradation in bit error rate than noise added in the channel since the clipping noise fades along with the signal.

\section{REPEATED FREQUENCY DOMAIN FILTERING AND CLIPPING RFC}

The proposed method is the same as previous method RCF, but with almost a simple change. The location of the filter becomes before the clipping as shown in figure 2, the frequency domain filtering that depends on the interpolation as noted by previous results that improve the BER but increases the PAPR

The basic idea of this method is that this filter will improve the performance of the OFDM to improve the BER and then the clipping will improves PAPR method is almost the same as RCF, the system have the same receiver But there is a difference in One block in the transmitter. This block is RFC as shown in figure 3 . Interpolated baseband signal followed by frequency domain filtering, the same filter which are explained in the case of RCF. The filtering signal is clipped in the time domain. The clipping block is described previously in the case of RCF. 


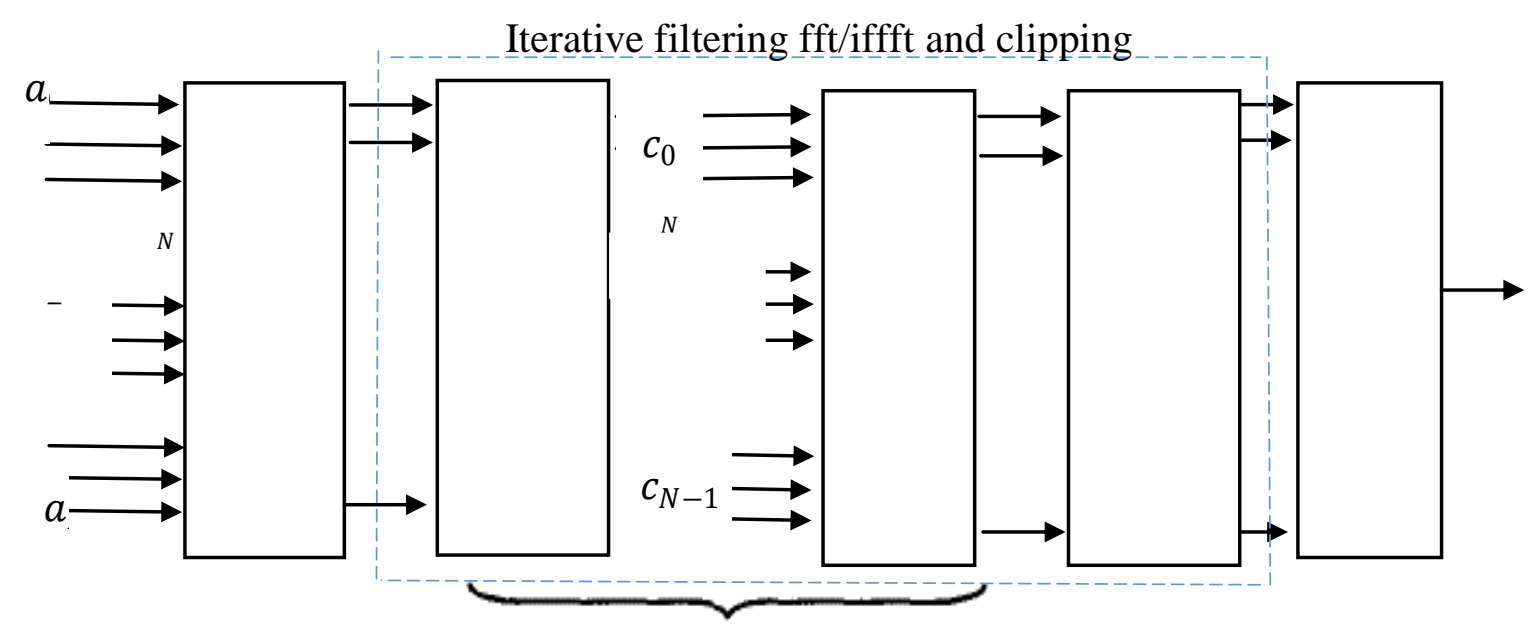

Figure 2 shows the block diagram of the new PAPR reduction scheme

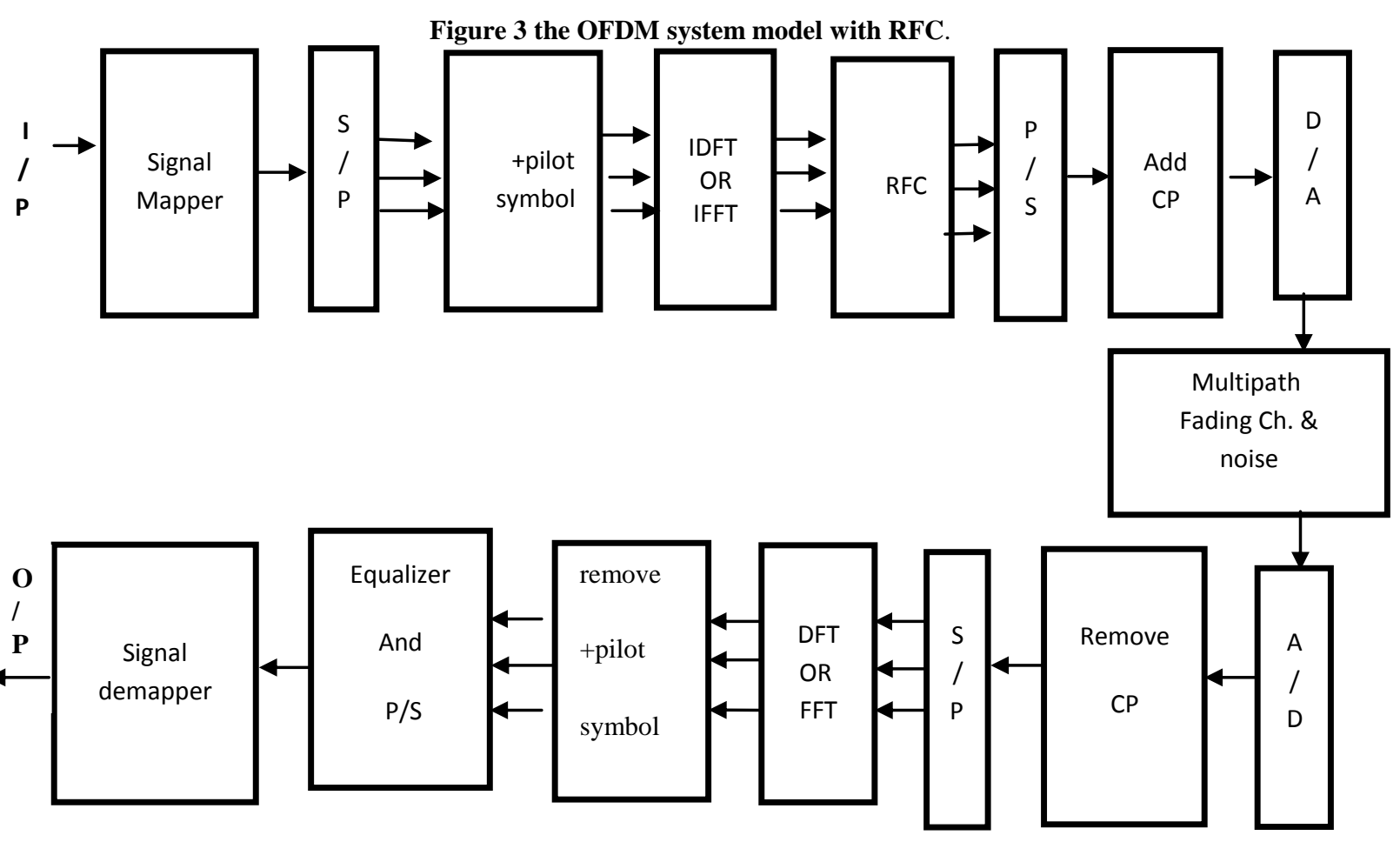

\section{SIMULATION RESULTS AND}

\section{ANALYSIS}

The system model used in the work is shown in figure 4.1. The OFDM parameter used in the test is the LTE parameters as shown in table 4.1
Table 4.1 LTE parameter

\begin{tabular}{|l|l|}
\hline FFT size =no. of frame & 128 \\
\hline frequency Spacing & $15 \mathrm{KHz}$ \\
\hline BW & $1.25 \mathrm{MHz}$ \\
\hline CP & 32 \\
\hline No of symbol & 1000 \\
\hline Sampling frequency & $192 \mathrm{MHz}$ \\
\hline Modulated type & QPSK \\
\hline
\end{tabular}


one can conclude From figure 4 and 5 the following:

There is an obvious improvement in the CCDF of PAPR and the BER of the RFC In comparison with the RCF.

The CCDF of PAPR of the RFC at $\mathrm{CR}=2$ is better than the CCDF of PAPR of the RCF at $C R=1.5$, in addition to that the BER of the RFC at $C R=2$ is better than the BER of the RCF at $\mathrm{CR}=1.5$.

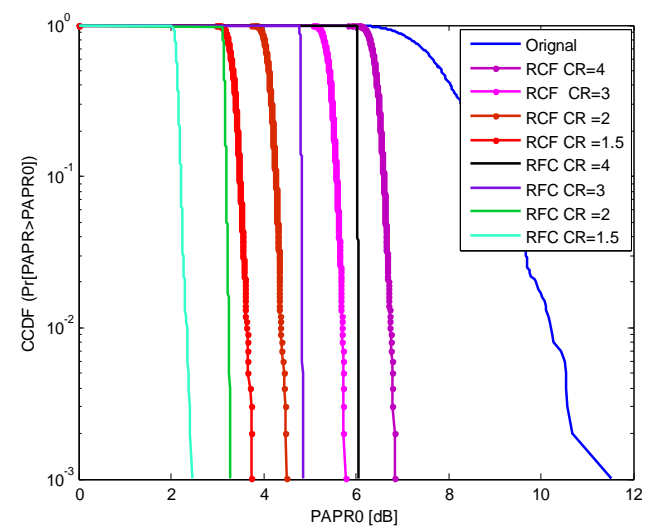

Figure 4 Comparison in CCDF of PAPR between RCF and $\mathrm{RFC}$, where $\mathrm{I}=4$

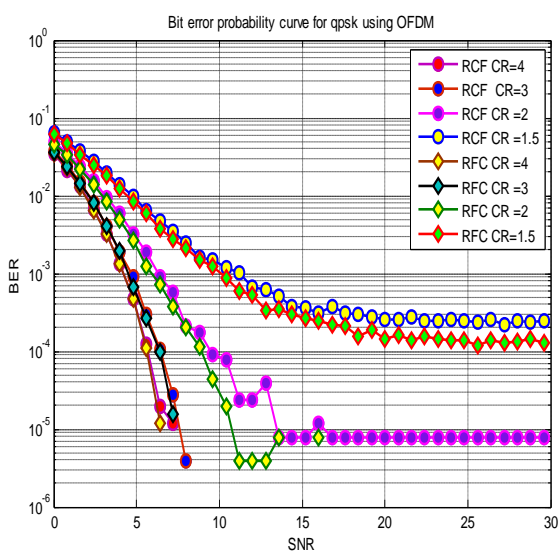

Figure 5 Comparison in BER between RCF and RFC, where $\mathrm{I}=4$.

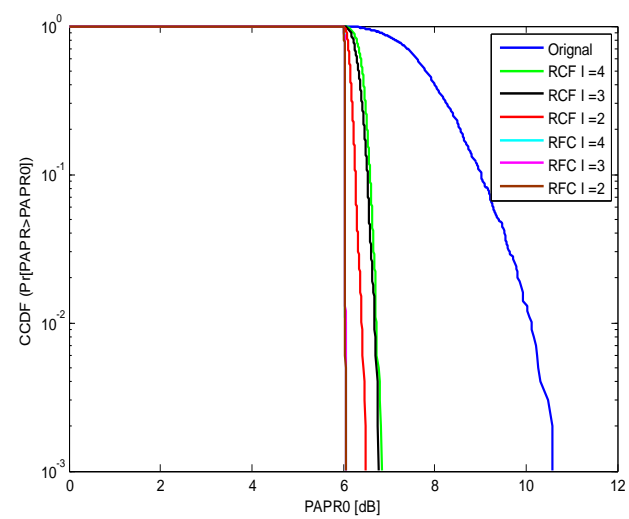

Figure 6 Comparison in CCDF of PAPR between RCF and $R F C$ where $C R=4$

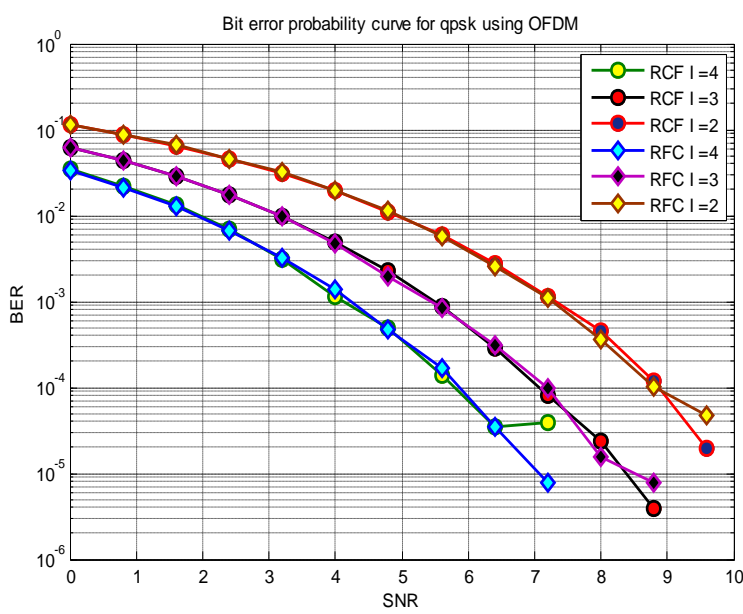

Figure 7 Comparison in CCDF of PAPR between RCF and $\mathrm{RFC}$ where $\mathrm{CR}=4$

As shown in figure 6 and 7 The CCDF of PAPR and The PAPR for I (N) was increased only Avery small amount could be neglected in comparison with I1.The BER decreases whenever I increased

The improvement in the PAPR at RFC can be seen when comparing figure

CCDF of PAPR was improved in all cases. The improvement ratio was increased with the decrease of $\mathrm{CR}$ and the increase of $I$. The biggest improvement is in the case ( $\mathrm{I}=3$ and $\mathrm{CR}$ $=4)$.

BER was improved for $(\mathrm{I}=3$ and $\mathrm{I}=4$ in all cases of $\mathrm{CR})$ and for $\mathrm{I}=2$ except when $(\mathrm{CR}=2)$ as for the rest I,BER sometimes better, sometimes worse, but by asmall ratio.

RFC is better than RCF because when I increase the BER improve and

PAPR almost preserves its value.

\section{CONCLUSIONS}

The RFC and RCF can improve the PAPR and BER at the same time.

RFC is better than RCF in performance especially when $\mathrm{I} \geq 2$ while maintaining the complexity and price of RCF.

RFC have the same complexity and cost RCF because RFC has not added a new function for RCF but the only change filter location.

\section{REFERENCES}

[1] K. Fazel and S. Kaiser, Multi-Carrier and Spread Spectrum Systems: From OFDM and MC-CDMA to LTE and WiMAX, 2nd Edition, John Wiley \& Sons, 2008.

[2] G. Wunder, R. F. H. Fischer, H. Boche, S. Litsyn, J,-S. No, "The PAPR Problem in OFDM Transmission: New Directions for a Long-Lasting Problem," IEEE Signal Processing Magazine, vol. 20, no. 6, pp. 130-144, November 2013. 
[3] T. Jiang, and Y. Wu "An Overview: Peak-to-Average Power Ratio Reduction Techniques for OFDM Signals" IEEE Transactions on Wireless Communications, vol.54, no2, pp.257 - 268. June 2008.

[4] M. M. Mowla, and S.M. M. Hasan "Performance Improvement of PAPR Reduction for OFDM Signal In LTE System," International Journal of Wireless \& Mobile Networks, vol. 5, no. 4,pp. 35-47,August 2013.

[5] C. Choudhary, and V. Gupta "A Study of Performance Enhancement Schemes for Multicarrier Transmission," International Journal of Computer Applications, vol. 68, no. 5, pp. 50-54, April 2013.

[6] H. D. Joshi. "Performance augmentation of OFDM system," Ph.D. dissertation, Jaypee Univ. of engineering and Technology, India, May 2012.

[7] Y. S. Cho, J. Kim, W. Y. Yang and C. G. Kahn, MIMO OFDM wireless communications with Matlab. Asia: John Wiley \& Sons, 2010.

[8] ITU-R, Report M.2134, "Requirements related to technical performance for IMT-Advance Radio Interface,"2008.

[9] R. Prasad. OFDM for Wireless Communications Systems. Artech House Publishers, August 2004.
[10] J. Armstrong. "New OFDM Peak-to-Average Power Reduction Scheme," IEEE VTS 53rd Vehicular Technology Conference, Spring, vol. 01, pp. $756-760$, May 2001.

[11] B. Schmidt, C. Ng, P. Yien, C. Harris, U. Saripalle, A. Price, S. Brewer, G. Scelsi, R. Slaviero and J. Armstrong' "Efficient Algorithms for PAPR Reduction in OFDM Transmitters Implemented using Fixed-Point DSPs," IEEE 63rd Vehicular Technology Conference, 2006, vol. 4 ,pp. $2023-2027$.

[12] J. Armstrong "Peak-to-Average Power Reduction in Digital Television Transmitters," Digital Image Computing Techniques and Applications, pp.1-6, January 2002.

[13] J. Armstrong. "Peak-to-average power reduction for OFDM by repeated clipping and frequency domain filtering," IEEE Electronics Letters, vol. 38, pp. 246 247, Feb 2002.

[14] C. A. Devlin, A. Zhu, and T.J. Brazil "Peak to Average Power Ratio Reduction Technique for OFDM Using Pilot Tones and Unused Carriers," Radio and Wireless Symposium, 2008 IEEE, pp. 33 - 36, Jan. 2008 